\title{
PAPER
}

\section{Tracing children's vocabulary development from preschool through the school-age years: an 8-year longitudinal study}

\section{Shuang Song, ${ }^{1}$ Mengmeng Su, ${ }^{1}$ Cuiping Kang, ${ }_{4}^{1}$ Hongyun Liu, ${ }^{2}$ Yuping Zhang, ${ }^{1,3}$ Catherine McBride-Chang, ${ }^{4}$ Twila Tardif, ${ }^{5}{ }^{\prime}$ Hong Li, ${ }^{2}$ Weilan Liang, ${ }^{6}$ Zhixiang Zhang $^{6}$ and Hua Shu ${ }^{1}$}

1. State Key Laboratory of Cognitive Neuroscience and Learning, Beijing Normal University, China

2. School of Psychology, Beijing Normal University, China

3. Department of Psychology, Chengdu Medical College, China

4. Department of Psychology, The Chinese University of Hong Kong, Hong Kong

5. Department of Psychology and Center for Human Growth and Development, University of Michigan-Ann Arbor, USA

6. Peking University First Hospital, China

\begin{abstract}
In this 8-year longitudinal study, we traced the vocabulary growth of Chinese children, explored potential precursors of vocabulary knowledge, and investigated how vocabulary growth predicted future reading skills. Two hundred and sixty-four (264) native Chinese children from Beijing were measured on a variety of reading and language tasks over 8 years. Between the ages of 4 to 10 years, they were administered tasks of vocabulary and related cognitive skills. At age 11, comprehensive reading skills, including character recognition, reading fluency, and reading comprehension were examined. Individual differences in vocabulary developmental profiles were estimated using the intercept-slope cluster method. Vocabulary development was then examined in relation to later reading outcomes. Three subgroups of lexical growth were classified, namely high-high (with a large initial vocabulary size and a fast growth rate), low-high (with a small initial vocabulary size and a fast growth rate) and low-low (with a small initial vocabulary size and a slow growth rate) groups. Low-high and low-low groups were distinguishable mostly through phonological skills, morphological skills and other reading-related cognitive skills. Childhood vocabulary development (using intercept and slope) explained subsequent reading skills. Findings suggest that language-related and reading-related cognitive skills differ among groups with different developmental trajectories of vocabulary, and the initial size and growth rate of vocabulary may be two predictors for later reading development.
\end{abstract}

\section{Introduction}

Children's oral vocabulary knowledge is an important indicator of language development and subsequent reading success or failure during formal schooling (Lee, 2010; Storch \& Whitehurst, 2002). Several studies have attempted to capture individual differences in the timing and rate of vocabulary development using a longitudinal approach, but most have traced such development for toddlers (before 46 months) over a relatively short period of time (Huttenlocher, Haight,
Bryk, Seltzer \& Lyons, 1991; Pan, Rowe, Singer \& Snow, 2005; Rowe, Raudenbush \& Goldin-Meadow, 2012; Vagh, Pan \& Mancilla-Martinez, 2009). However, children's oral vocabulary continues to grow rapidly and to overlap with reading acquisition in primary school. Understanding the path of children's vocabulary growth after early childhood and its associated potential precursors has both theoretical and applied relevance for instructional approaches. The primary focus of this 8-year longitudinal study was to trace trajectories of Chinese children's oral vocabulary

Address for correspondence: Hua Shu, State Key Laboratory of Cognitive Neuroscience and Learning, Beijing Normal University, 100875, China; e-mail: shuh@bnu.edu.cn

Shuang Song and Mengmeng Su contributed equally to this work. 
development from ages 4 to 10 years old in mainland Chinese children.

In Perfetti's DVC (Decoding, Vocabulary, and Comprehension) triangle model (Perfetti, 2009), vocabulary knowledge falls between word identification and reading comprehension (Perfetti, Landi \& Oakhill, 2005). Multiple studies have supported this view by showing a close relationship between vocabulary and word reading or reading comprehension (Nation, Cocksey, Taylor \& Bishop, 2010; Zhang, Tardif, Shu, Li, Liu, McBrideChang, Liang \& Zhang, 2013; Zhang, McBride-Chang, Wong, Tardif, Shu \& Zhang, 2013). In a recent longitudinal study of 262 Chinese children, Zhang, Tardif et al. (2013) found that children's vocabulary abilities at age 4 and age 5 mediated the effects of SES on children's word reading ability in the third grade. At a higher-order level of reading, children's vocabulary knowledge at ages 6,7 , and 8 can discriminate typically developing controls from poor reading comprehenders in both English and Chinese (Nation et al., 2010; Zhang, McBride-Chang et al., 2013). There is likely a bidirectional relationship (vocabulary-reading development) year by year. Children learn more vocabulary through reading, while vocabulary knowledge also helps children to read. A second goal of the present study, therefore, was to explore the relationship between natural vocabulary development and later reading skills.

Previous studies have often focused on vocabulary growth of toddlers or children in early childhood. These studies have typically used receptive picture naming tasks to measure children's vocabulary breadth (Huttenlocher et al., 1991; Rowe et al., 2012; Vagh et al., 2009). Children's vocabulary ability continues to develop rapidly after they enter school, and large individual differences in vocabulary knowledge have been observed. Given this situation, Perfetti (2009) suggested that variance in knowledge of word meaning might be taken into consideration for older children. In order to detect lexical development both before and after the beginning of literacy skills, in the present study we chose an expressive vocabulary definition measure. This measure focused on children's knowledge of word meanings and has been used in a number of previous studies as a reasonable indicator of Chinese children's vocabulary development from ages 4 to 9 (Chow, McBride-Chang \& Burgess, 2005; Lei, Pan, Liu, McBride-Chang, Li, Zhang, Chen, Tardif, Liang, Zhang \& Shu, 2011; McBride-Chang, Chow, Zhong, Burgess \& Hayward, 2005b; McBrideChang, Liu, Wong, Wong \& Shu, 2011; McBride-Chang, Shu, Zhou, Wat \& Wagner, 2003; McBride-Chang \& Ho, 2005; McBride-Chang, Tardif, Cho, Shu, Fletcher, Stokes, Wong \& Leung, 2008; Pan, McBride-Chang, Shu, Liu, Zhang \& Li, 2011).
There is consensus that substantial individual differences exist in the rate and shape of vocabulary growth. Vocabulary varies widely in the initial size and growth rates between children; some start slow and speed up, while others start fast and continue at a steady pace (Rowe et al., 2012). Therefore, initial size and developmental speed may be two meaningful indices of vocabulary growth. In one study, individual differences in initial vocabulary size were observed for both L1 and L2 learners at age 7; such differences could predict the subsequent growth of reading comprehension skills (Lervåg \& Aukrust, 2010). Moreover, modeling individual growth rates can provide a sensitive measure of differences between catch-up versus delayed groups and can determine which factors best predict patterns of such group differences (Singer \& Willett, 2003). For example, Fernald and Marchman (2012) found that late talkers with efficiency in lexical processing at 18 months showed more accelerated vocabulary growth over the following year compared with late talkers who were less efficient in early speech processing. They also divided children's vocabulary development into four subgroups demonstrating different initial vocabulary size and rate. Such clustering analyses help to clarify the extent to which subgroups of children with different developmental patterns can be identified using early cognitive skills (Boscardin, Muthén, Francis \& Baker, 2008). By using a data-driven approach, we sought to chart individual variation in both initial vocabulary size and growth rate and establish subgroups of lexical growth over a long period of time. In particular, we wanted to determine whether children with low vocabulary knowledge at age 4 would remain delayed after they entered primary school or, alternatively, would catch up with development.

Individual differences in children's vocabulary growth can be explained in terms of experience-based factors, such as family socioeconomic status (SES) (Hoff, 2003; Rowe \& Goldin-Meadow, 2009; Rowe et al., 2012), parent language input (Hurtado, Marchman \& Fernald, 2008; Pan et al., 2005) and home literacy (Duursma, Romero-Contreras, Szuber, Proctor, Snow, August \& Calderón, 2007). Studies across languages have also demonstrated that children's early vocabulary growth is associated with certain cognitive skills, such as working memory, phonological processing skills and morphological awareness (Bowey, 2001; Gathercole, Willis, Emslie \& Baddeley, 1992; Just \& Carpenter, 1992; McBrideChang, Wagner, Muse, Chow \& Shu, 2005c; Stokes \& Klee, 2009). In the present study, we tested whether and how children's initial vocabulary and vocabulary growth are affected by both environmental factors and these cognitive skills. Because vocabulary and reading development overlap after children enter school, we also 
included some other reading-related cognitive factors, including rapid automatized naming (Pan et al., 2011) and orthographic awareness (Ho, Chan, Lee, Tsang \& Luan, 2004; Li, Shu, McBride-Chang, Liu \& Peng, 2012; Shu \& Anderson, 1998). We thus investigated whether and how environmental and cognitive factors might jointly account for individual differences in Chinese children's vocabulary development.

The role of vocabulary knowledge in reading development has attracted much attention in recent years. Perfetti's Decoding, Vocabulary, and Comprehension triangle model emphasizes the central role of vocabulary by assuming that it has respective reciprocal relations with both decoding and comprehension (Perfetti, 2009). This theoretical explanation has led to many studies focusing on the relations between vocabulary growth and reading development (Lee, 2010; Verhoeven, van Leeuwe \& Vermeer, 2011; Zhang, Tardif et al., 2013). The present longitudinal study allowed us to investigate the relationship between vocabulary growth and later reading skills. One recent study showed that compared to estimates of initial size, the estimated rate and/or acceleration in vocabulary growth between 14 and 46 months could better predict later vocabulary size at 54 months (Rowe et al., 2012). In the present study, we wanted to determine if the rate and/or acceleration in vocabulary growth of older children could also explain later reading performance. By using an intercept-slope cluster method, we investigated how the growth rate of children's vocabulary from ages 4 to 10 and the initial vocabulary size explained later reading skills at age 11 .

Three research questions were addressed in the present study. First, could we describe a long-term dynamic change in vocabulary growth and separate the subgroups from age 4 to age 10 ? Second, and more specifically, what are the associated factors, particularly cognitive skills, behind different vocabulary development profiles? Finally, to what extent can original vocabulary and lexical growth rate from age 4 to age 10 predict reading performance at age 11 ?

\section{Method}

\section{Participants}

In this 8-year longitudinal study, 264 typically developing Chinese children (145 boys and 119 girls) were included from a longitudinal study of language and literacy Chinese Communicative Development Inventory (CCDI; Tardif, Fletcher, Zhang \& Liang, 2008). This study has been ongoing since 2000 (Lei et al., 2011; Pan et al., 2011). Initially, 338 children with normal IQs were recruited from the longitudinal study of language and literacy. Thanks to the cooperation of parents, the attrition rate was low, with 293 from the initial sample continuing to participate in the project at present. In the present study, we report on data from 264 children who entered school in the same year. All 264 children were native Mandarin speakers from Beijing, China, with their first language being Mandarin Chinese. All of these children have been tested annually on a variety of tasks since 2001 with an interval of approximately 12 months. There were virtually no missing data, with no more than three missing values on several measures. The present study focused on the vocabulary skills and language- and reading-related cognitive skills, so we selected the data for those from 4.4 years old to 11.4 years old. Informed consent was obtained from the parents. Table 1 shows the details of each task across years.

\section{Measures}

\section{Vocabulary definitions}

This Vocabulary definitions task was used for children from age 4 to age 10. From age 4 to age 8, 32 words representing concepts or objects arranged in order of increasing conceptual difficulty and with decreasing word frequency were orally presented to children. Children were required to provide the definition for each word. This task was translated and adapted for Mandarin Chinese-speaking children from the vocabulary subset of the Stanford-Binet Intelligence Scale (Thorndike, Hagen \& Sattler, 1986). It was similar to those used in previous studies (e.g. Anglin, 1993; Gathercole, Service, Hitch, Adams \& Martin, 1999; Lervåg \& Aukrust, 2010). For instance, the experimenters asked a child 'What is a kitchen?' Scoring was based on the number of important semantic features included, following the scoring scheme in the test manual. The ('well-formed-ness') form of the definition (syntactic ability) was not considered in the marking process. One point was given for each feature with a maximum score of 2 for each item. A complete definition had to include the proper semantic category and one or more features. For example, for the target word kitchen, a 2-point answer is 'a place for cooking'. A 1-point answer is 'a place at home'. An example of a zero-point answer would be that the child would simply repeat the word again. Examples of tested words and the model answers with key features are available upon request from the authors. The task was stopped when the child obtained zero marks across five consecutive items; the maximum score of the task was 64 . Children's responses were rated by 
Table 1 Raw mean, standard deviation, reliability, skewness, and kurtosis statistics for different tasks

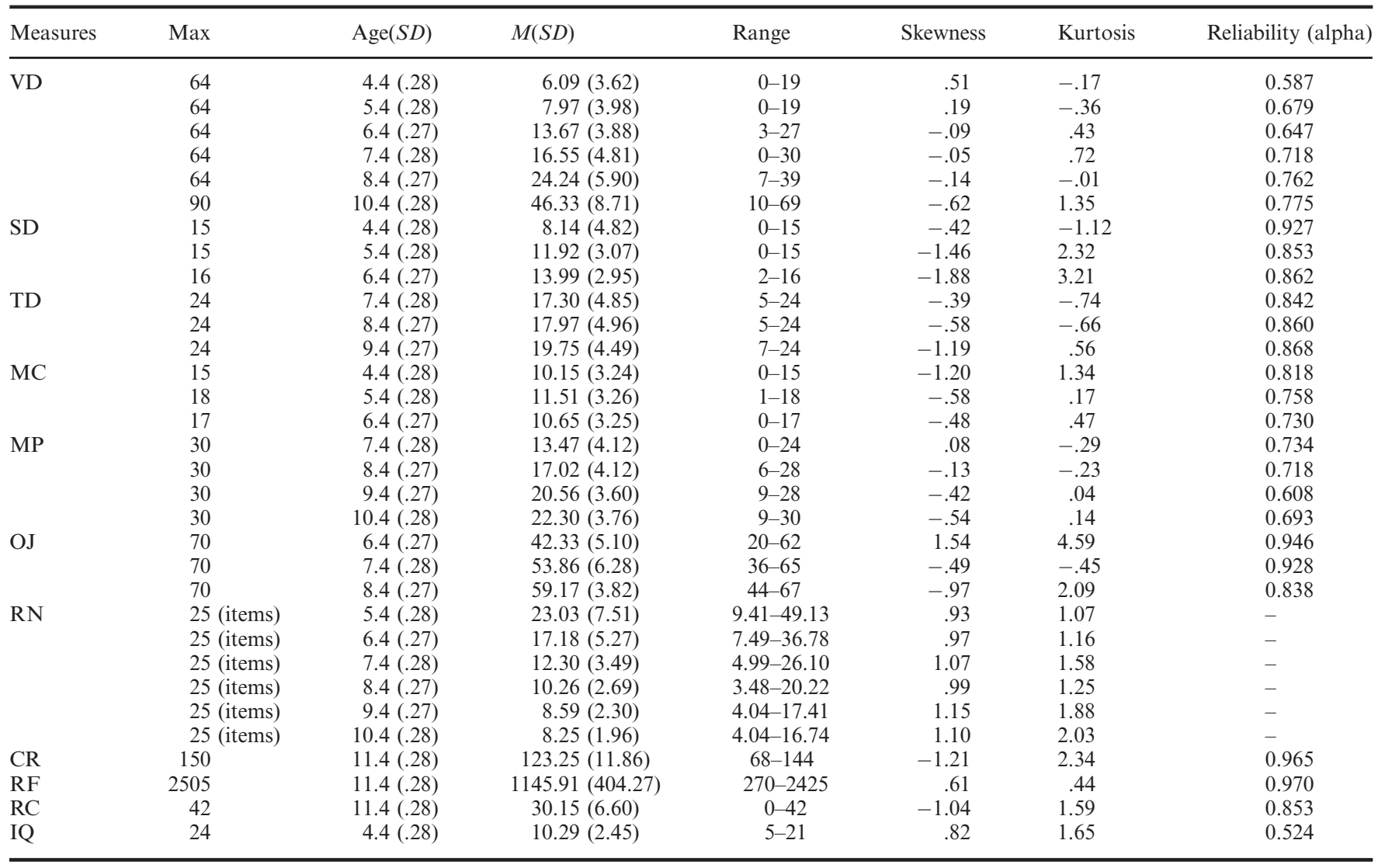

Note 1: $\mathrm{VD}=$ Vocabulary Definition, $\mathrm{SD}=$ Syllable Deletion, $\mathrm{TD}=$ Tone Detection, $\mathrm{MC}=$ Morphological Construction, $\mathrm{MP}=\mathrm{Morphological}$ Production, OJ = Orthographic Judgment, RN = Rapid Digital Naming, CR = Character Recognition, RF $=$ Reading Fluency, RC $=$ Reading Comprehension, IQ = Nonverbal IQ.

Note 2: Data are reported for 264 children who entered school in the same year with no more than three missing values on some measures.

two trained experimenters with high inter-rater reliability during pilot tests. This task has been used in previous studies and has been suggested to be a reasonable proxy for vocabulary knowledge (Chow et al., 2005; Lei et al., 2011; Liu \& McBride-Chang, 2010; McBride-Chang, Cho, Liu, Wagner, Shu, Zhou, Cheuk \& Muse, 2005a; McBride-Chang et al., 2005b; McBride-Chang et al., 2011; McBride-Chang et al., 2003; McBride-Chang et al., 2005c; McBride-Chang et al., 2008; Pan et al., 2011; Tong, McBride-Chang, Shu \& Wong, 2009; Zhang, Tardif et al., 2013). To avoid a ceiling effect, the age-10 version included an additional 13 items from the Hong Kong version. Because we tapped a variety of children's cognitive abilities every year in this longitudinal study, the measures administered each year were not necessarily the same. When the children were age 9, we added some more tests tapping children's attention. Given that the testing time for a child had to be limited to 2 hours or less in order to avoid tiredness of the children, we did not measure vocabulary knowledge of the children at age 9 .

Phonological awareness

Syllable deletion. Used for children between ages 4 and 6 (Lei et al., 2011), this task asked children to delete one syllable from two or three syllable phrases (e.g. /hong2 yan2 se4/, meaning red color, with the syllable /hong2/ taken away would be /yan2 se4/, meaning color).

Tone detection. Used between ages 7 and 9, the tone detection task is closely related to the tone awareness task used by Chen, Anderson, Li, Hao, Wu and Shu (2004). It consists of 12 syllable trials and 12 non-syllable trials. Children were presented with three syllables and asked to identify one syllable with a different tone from the other two syllables (e.g. the tone that is different from the other two among the following three syllables, /kui2/ 
meaning sunflower, /bo2/ meaning thin, and /ban1/ meaning class, is /ban $1 /$ ).

Morphological awareness

Morphological construction. Following previous work (McBride-Chang et al., 2003), in this task, children were required to construct a new compound word with the morphemes they had previously acquired. For instance, the experimenter asked, "A piece of paper with white color is called white paper /bai2 zhi3/; what would it be called if the color of the paper was red?' The right answer in this case is 'red paper' /hong2 zhi3/. This task was used from ages 4 to 6 years.

Morphological production. In this task, children listened to a compound word composed of two morphemes. They were asked to produce two new words with the target morpheme (e.g. one target morpheme was /baol/ meaning bag from /pi2 baol/ meaning leather bag). The morpheme in one of these two words was required to have the same meaning as that of the target morpheme (e.g. the $/ \mathrm{bao} 1 /$ in $/ \mathrm{shou} 3 \mathrm{bao} 1 /$ meaning hand bag, has the same meaning as the /baol/ in /pi2baol/), while the morpheme in the other word has a different meaning from the target morpheme (e.g. in the word /mian4 baol/ meaning bread, /baol/ has to do with bread, rather than a bag). This task has been successfully used in a previous study (Shu, McBride-Chang, Wu \& Liu, 2006) and was used for ages 7 to 9 .

\section{Orthographic judgment}

This task was used for children from age 6 to age 8 . Shu and Anderson (1998) created this orthographic judgment task to measure the orthographic awareness of Chinese children. It has been used in previous research as a reasonable index for orthographic awareness ( $\mathrm{Li}$ et al., 2012; Zhang, Tardif et al., 2013). In this lexical decision task, children were visually presented with 70 items consisting of line drawings, non-characters and real characters. They were asked to judge whether the item was a real character or not. One point was obtained for a right answer, so the total possible score was 70 . Of the 70 items, 10 were black-and-white line drawings with no conventional stroke patterns (e.g. 田). The other 20 items included two types of compound non-characters: 10 were ill-formed structure non-characters with real radicals in illegal positions (e.g. 呫) and 10 were ill-formed radical non-characters which looked like standard compound characters with the radical in a legal position (e.g. II). In addition, there were 10 well-formed structure and radical pseudo-characters, which were not real characters in the

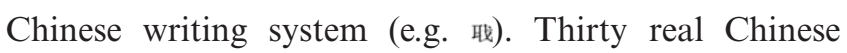
characters were also included as fillers.

\section{Rapid digit naming}

Used from age 5 to age 10, rapid digital naming consisted of 5 different digits repeated randomly five times (Lei et al., 2011; Pan et al., 2011). The children's task was to name the digits as accurately and as quickly as possible twice. Mean time in seconds was calculated.

\section{Character recognition}

This task was measured at age 11. It comprised 150 characters of increasing difficulty level. Children were asked to name the characters without a time limit. All of these particular characters are required to be learned in China by the end of primary school (Shu, Chen, Anderson, Wu \& Xuan, 2003). The character recognition task has been widely used as an indicator for Chinese children's literacy skills (Lei et al., 2011; McBrideChang et al., 2003).

\section{Reading fluency}

Following the procedures of previous research (Moll, Fussenegger, Willburger \& Landerl, 2009; Pan et al., 2011), children were asked to silently read 100 sentences in 3 minutes and to evaluate whether each sentence was literally correct or not. Characters in corrected sentences per minute were totaled for the child's final score. This task was measured at age 11 .

\section{Reading comprehension}

This task was measured at age 11. Four passages and 42 multi-choice questions were given. In each question, children were asked to choose one from the four choices that would best answer the question. Answers ranged from single words to phrases or sentences.

\section{Nonverbal IQ}

This task was measured at age 4, and only the first two sets from Raven's Standard Progressive Matrices (Raven, Court \& Raven, 1996) were chosen.

\section{Mother's education level}

Mothers of the participants were invited to complete the assessment (Zhang, Tardif et al., 2013). The choices for the 7-point self-report scale were $1=$ lower than third grade, $2=$ fourth to sixth grade, $3=$ junior high school 
completion, $4=$ senior high school completion, $5=$ college ( 3 years), $6=$ university graduate ( 4 years), and $7=$ graduate school. In China, college refers to the 3-year college or so-called 'da zhuan'. The term 'university graduate' refers to a 4-year undergraduate education. In China, students who achieve a better performance will go to the 4-year undergraduate university, while others may go to the 3-year 'da zhuan'.

\section{Statistical approach}

In this study, we were interested in describing children's individual differences and distinguishing heterogeneous subgroups in vocabulary growth based on our longitudinal data from age 4 to age 10 years. A two-step process was used to identify the subgroups with different initial size and pace of vocabulary development (Tataryn \& Chochinov, 2002).

In our first step, for each participant $i$ at time $t$, linear growth models were used to transform scores of participants into two parameters, the slope and the intercept (Rogosa, Brandt \& Zimowski, 1982):

$$
\mathrm{y}_{\mathrm{i}}(\mathrm{t})=\delta_{0 \mathrm{i}}+\delta_{1 \mathrm{i}}\left(\mathrm{t}-\mathrm{t}_{0}\right)+\mathrm{e}_{\mathrm{it}}, \quad \mathrm{e}_{\mathrm{it}} \sim \mathrm{N}\left(0, \sigma_{\mathrm{i}}^{2}\right)
$$

where $y_{i}(t)$ is the vocabulary score of participant $i$ at time $\mathrm{i}, \delta_{0 \mathrm{i}}$ is the estimate of intercept for participant $\mathrm{i}, \delta_{0 \mathrm{i}}$ is the estimate of slope for participant $\mathrm{i}$, and $\mathrm{t}_{0}$ is the youngest age of all participants in the first measure. Here $e_{i t}$ means residual error. The individual data were transformed by using the MATLAB software 'polyfit' function. The ordinary least squares method (OLS)made it reliable to estimate the parameters for participants with missing data. Since children's development appeared to be relatively steady, these estimates, including six time points, could well explain their development.

In our second step, a clustering nearest centroid sorting method was used with the fastclus procedure with the Statistical Analyses Software (SAS). The main idea for the clustering was to classify several similar participants into one subgroup. In the cluster procedure, two statistical indices were considered to determine the number of groups, namely the $R$-square and the Pseudo- $F$ statistics. The larger the $R$-square, the better the groups clustered. Larger Pseudo- $F$ statistics were also considered better (Kale, 1995).

\section{Results}

Table 1 displays the means and standard deviations of all measures, as well as the skewness, kurtosis and reliability estimates of each task. Generally, most of the measures were normally distributed with relatively high reliabilities, and children's performance on the measures improved with age.

Based on vocabulary raw scores, clusters with between two and five classes were tried for the intercept and slope. The results showed that compared with other cluster solutions, the three-cluster model had the highest Pseudo$F$ statistics (172.00) and responsible $R$-square statistics (0.67). Therefore, the three-cluster model provided the most meaningful and concise fit for the data. Figure 1(A) presents the scatter plot of intercepts and slopes for our three subgroups. The first subgroup $(n=85)$, called the high-high group, was characterized by a high intercept (4.72) and a high slope (6.80). The second $(n=137)$, called the low-high group, showed a low intercept $(-.60)$ but a high slope (7.05). The third $(n=42)$, termed the low-low group, had both a low intercept (2.55) and a low
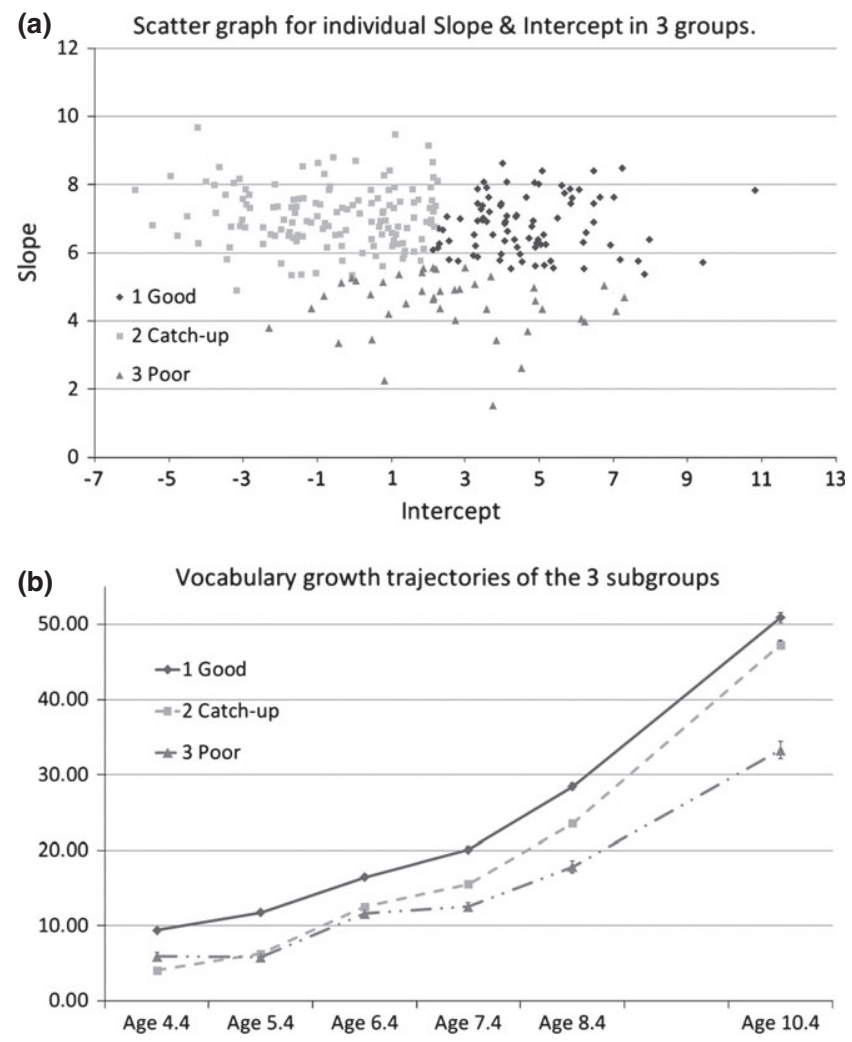

Figure 1 Scatter graph and vocabulary growth trajectories of the three subgroups.

Note 1: Intercept in figure (A) stands for the estimate of the vocabulary score at the youngest age of all participants for each child. Slope indicates the estimate of the growth of vocabulary scores per year for each child.

Note 2: At age 9, we added some more tests tapping children's attention. Given that the testing time for a child of necessity had to be limited to 2 hours to avoid fatigue effects, we did not measure vocabulary knowledge for age 9 children. 
slope (4.49). The mean scores of the vocabulary definition task across six time points for participants in the three clusters are plotted in Figure 1(B).

The means and standard deviations of the six time points' vocabulary scores, value of $F$, and comparisons (Tukey's post-hoc test) among the three groups are shown in Table 2. The results revealed that children in the high-high group performed significantly better than those in the low-high and low-low groups at 4 years old $(F(2,261)=97.54, p<.001)$, and the latter two groups did not differ significantly until age 7 . After entering school at age 7 , participants in the low-high group quickly became significantly better than those in the low-low group.

Table 2 also includes the means and standard deviations of other measures at different ages among the three trajectories. Children in the high-high group tended to have relatively higher IQs and mothers' education levels as compared to those in the low-high group, while there were no significant differences in these between the lowhigh and low-low groups, suggesting that children in the high-high group were apparently advantaged in both their IQ and in environmental conditions compared with the low-high and low-low groups.

Table2 Participants' performance over time among the three groups

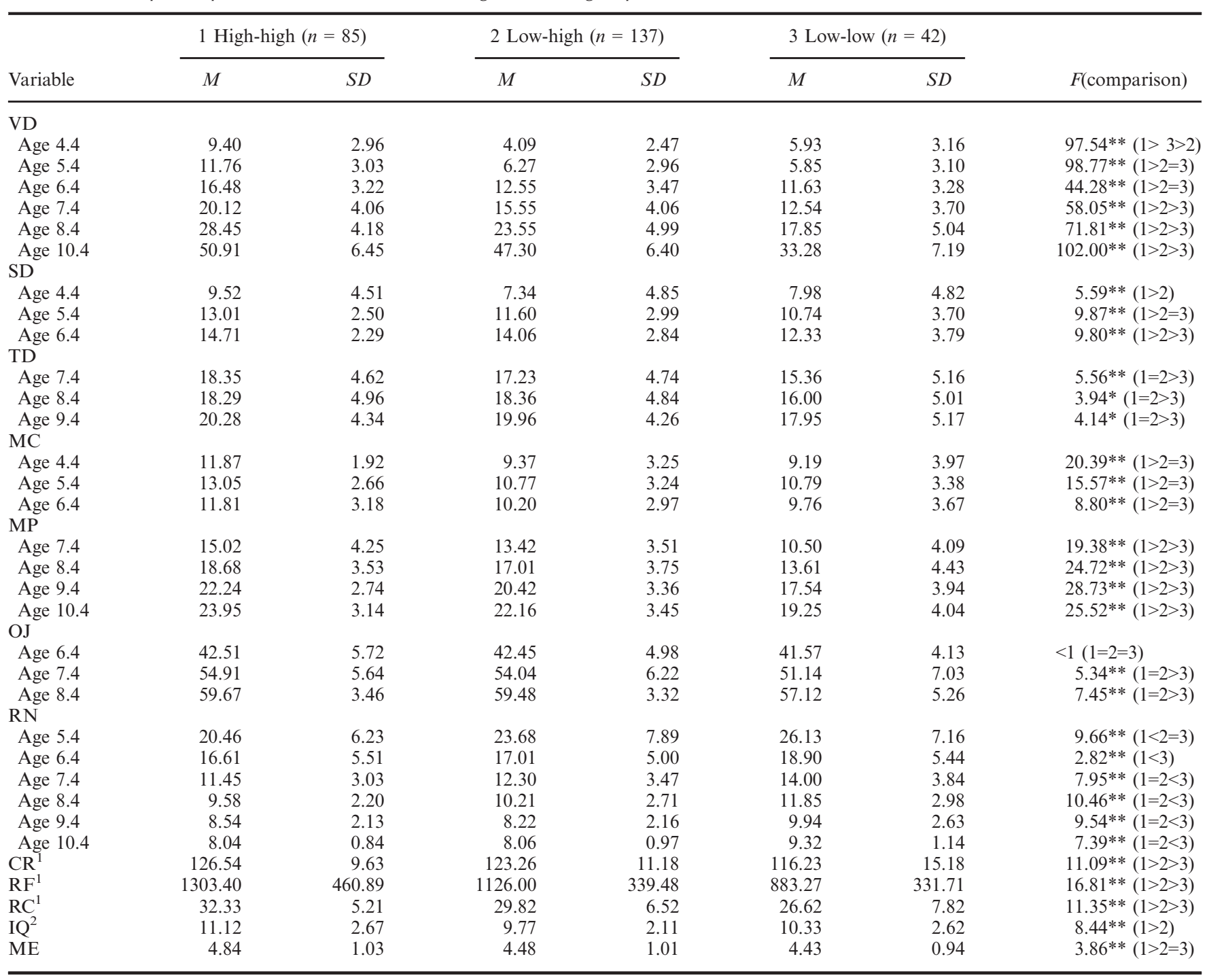

Note 1: $\mathrm{ME}=$ Mother's Education. ${ }^{1}$ Tested at age 11.4. ${ }^{2}$ Tested at age 4.4.

Note 2: Scores were calculated in seconds in the RN (Rapid Digit Naming task). Thus the quicker, the better. 
Table 2 further shows that children in the high-high group performed better than those in the low-low group on almost all tasks at all ages. However, for phonological and morphological skills, the low-high group performed differently at preschool and school ages. The low-high group performed similarly to the low-low group in preschool. However, this group differed significantly from the low-low group for syllable deletion at age 6 $(F(2,261)=9.80, p<.001)$ and on the tone detection task between age $7(F(2,261)=5.56, p=.004)$ and age 9 $(F(2,260)=4.14, p=.017)$. A similar pattern was found for the low-high group on morphological skills. The lowhigh group's scores on morphological construction were close to those of the low-low group in preschool but became significantly different from the low-low group on morphological production when children received formal education between ages 7 and 9. Detailed effect sizes are shown in Figure 2(A).

The same trend for the low-high group can also be found in rapid digit naming and orthographic judgment tasks in the primary school (Table 2). That is, for the orthographic tasks, although there were no significant

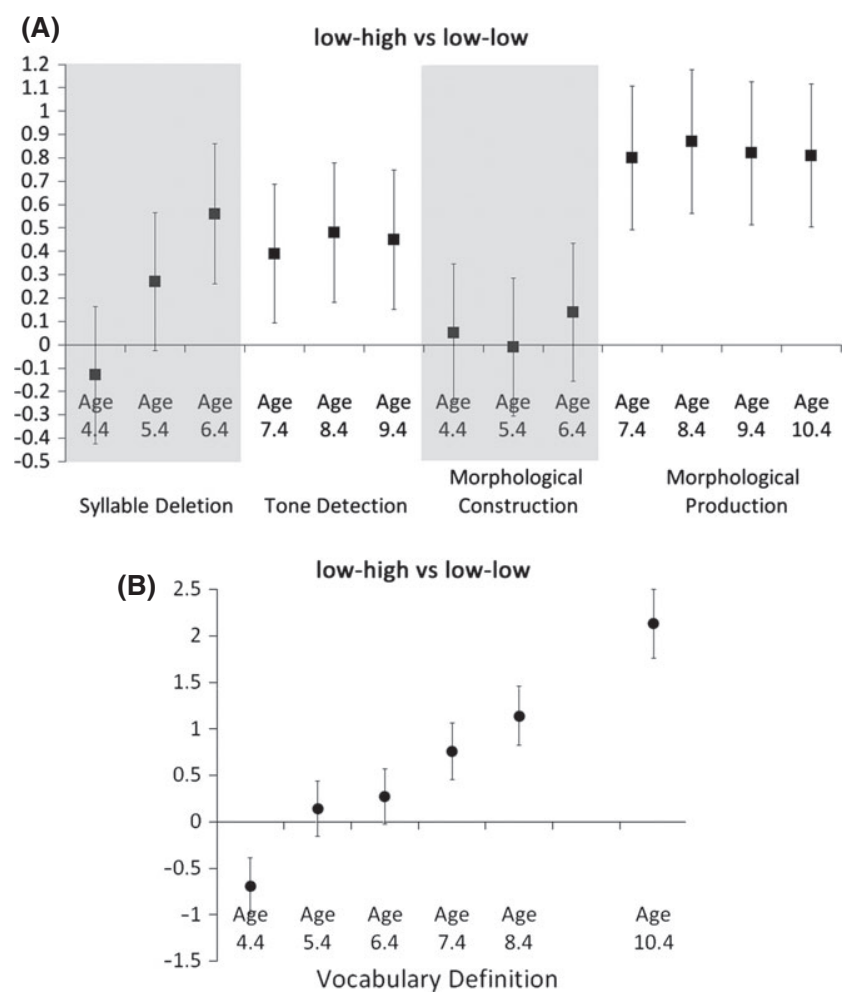

Figure 2 Effect size (Cohen's d) for Low-high and Low-low in phonological and morphological skills, as well as vocabulary definition test.

Note: Cohen's d is calculated using pooled standard deviations. differences among the three groups at age 6, the low-high group's scores were significantly higher than those of the low-low group and similar to those of the high-high group at ages 7 and 8 . A similar pattern was found for rapid digit naming.

Overall, then, the low-high group performed similarly to the low-low group across most cognitive tasks in preschool but became significantly higher than the lowlow group and on a par with the high-high group after entering school; in contrast, the high-high and low-low groups were consistent in their performances across tasks throughout childhood. This pattern reflects the vocabulary profiles themselves among the three groups over the same period in Figure 2(B).

Finally, these groups could also discriminate the three reading outcomes at 11 years old. To assess the predictive power of vocabulary growth for subsequent reading skills, correlations among mother's education level, nonverbal IQ, vocabulary intercept, vocabulary slope and reading skills (reading comprehension, reading fluency and character recognition) were calculated. Mothers' education levels, non-verbal IQ, vocabulary intercepts and slopes were all significantly correlated with the three reading outcomes (Table 3 ).

Hierarchical regression models further showed that controlling for mothers' education levels and non-verbal IQ, both vocabulary intercept and vocabulary slope from ages 4 to 10 uniquely explained the variations in reading comprehension, reading fluency and character recognition at age 11 . Together, they explained approximately $16.0 \%, 17.4 \%$ and $15.8 \%$ of the total variation in each, respectively (Table 4). Moreover, the intercept and slope variables could explain $10.6 \%, 9.4 \%$ and $7.3 \%$ of the variance in reading comprehension, reading fluency and character recognition separately even after statistically controlling early phonological skills, morphological skills, and rapid naming (Table 5).

\section{Discussion}

In this 8-year longitudinal study, we investigated vocabulary development of Chinese children in relation to reading from age 4 to age 11 . Three subgroups were clustered according to developmental trajectories of vocabulary growth (i.e. high-high group, low-high group, low-low group). The vocabulary knowledge of the high-high group was clearly distinguishable from those of both the low-high and low-low groups at age 4 . Vocabulary knowledge of the low-high group and the low-low group were comparable at the start, but they were distinguishable after these children entered primary school, despite sharing a similar background 
primary school and receiving formal reading instruction. Therefore, tracing children's vocabulary development from preschool to schooling is of great importance for understanding how family and school factors interact to influence children's lexical growth in the transition from pre-reader to reader. The present study has addressed this issue by modeling vocabulary growth in children from 4 to 10 years of age and distinguished three heterogeneous subgroups.

Our results showed that the high-high group performed better than the low-high and low-low groups in vocabulary and cognitive skills prior to school entry, presumably due to the relatively high level of their mothers' education. This finding is consistent with previous findings, suggesting that experience-related factors, such as mother's education in this study, may affect children's vocabulary growth in early childhood (Hoff, 2003; Rowe \& Goldin-Meadow, 2009; Rowe et al., 2012). The present study's novel finding is that though similar to the low-low group during preschool, children in the low-high group are comparable to the high-high group in vocabulary and cognitive skills after entering primary school. This finding indicates that children's vocabulary and cognitive skills are closely related, and formal schooling may make up for temporary weaknesses of low-high group children in both domains. The relationship between vocabulary and cognitive skills (shown in Table 2 and Figure 2(A) and 2(B)) found in the present study is also in line with those from previous studies (e.g. Bowey, 2001; McBride-Chang et al., 2005a).

Our results showed that vocabulary knowledge can predict later reading skills, including character recognition, reading fluency, and reading comprehension. To some extent, our findings lend support to Perfetti's (2009) DVC triangle model. Although there is substantial evidence showing the close relationship between vocabulary and reading skills, fewer studies have taken growth rate into consideration. Linear growth models have allowed us to identify the subgroups with different initial sizes and paces of vocabulary development. We found that both starting point and growth rate of vocabulary could predict a variety of later reading skills, including character recognition, reading comprehension and fluency.

Tracing children's vocabulary development over time and unraveling the longitudinal relations between vocabulary and reading skills may contribute to theories of Chinese literacy acquisition. In addition, the findings of the present study may shed light on educational practices relevant to early identification of children at risk for developing language and reading problems. Children who are relatively delayed in vocabulary knowledge in early childhood might still reach a normal level of vocabulary knowledge after they receive formal instruction. Early measures of both vocabulary and languagerelated cognitive skills are of great value for identifying children who are at risk for language and reading problems.

We are interested in the possible protective factors that enabled the low-high group to show faster growth on virtually all measures and overcome initial weaknesses. As shown in Figure 2(A), children's performance on phonological awareness between the low-high group and the low-low group began to be differentiated from age 5 . This difference continued and became larger at age 6 . We could speculate that the differences in phonological awareness between the low-high and low-low groups may help us to differentiate them even before school entry. So phonological awareness may be a possible preschool protective factor that enabled the low-high group to catch up. After entering school, the development of other cognitive skills, such as morphological awareness and rapid digit naming, are possible protective factors for the low-high group children. Moreover, perhaps dynamic response-to-intervention interactions can also accurately distinguish children who will be able to catch up once they receive adequate instruction versus those who will continue to struggle with language and reading. In the present study, we can only give some descriptive explanations of the factors underlying the differences between the low-high group and the low-low group. Further studies will be needed to fully understand the catch-up or fall-behind changes in children's vocabulary and reading development.

The work presented here had some limitations. First, due to the fact that we had relatively few measurement points for the regression, the estimates of all intercepts and slopes used in the developmental trajectories might have been slightly less reliable than would be optimal. Second, in the scatter graph for individual intercepts and slopes, there were relatively few participants with both not a very low intercept (i.e. a relatively good performance to begin with) and a low slope (falling behind), so they were classified into the low-low group due to their small number and the data-driven method. We did not attempt to understand the characteristics of these high-low group children because of their relatively low prevalence overall. Future research studies on larger samples will be needed to unravel the characteristics of these high-low group children and the reasons for their falling behind later. It should be noted that this study was only preliminary in tackling of the relations among vocabulary, cognitive skills, and reading skills. Because the relations among them are complex, they deserve further investigation in the future. 


\section{Conclusion}

This study explored the long-term vocabulary development of Chinese children from ages 4 to 10 years. Three growth profiles with different starting points and growth trajectories were observed, namely a high-high group, a low-high group and a low-low group. Familial factors and reading- or language-related cognitive skills were found to be associated with these developmental subgroups. Meanwhile, both the initial size and growth rate of vocabulary from ages 4 to 10 could well predict children's reading level in fifth grade. These results highlight the importance of focusing on the combination of intercepts and slopes for understanding lexical development. Our findings on the characteristics of the lowhigh group may stimulate educators to explore better instruction for at-risk children.

\section{Acknowledgements}

This research was supported by the National Key Basic Research Program of China (2014CB846103), by the Key Project of Philosophy and Social Sciences Research, Ministry of Education (11JZD041), by the Natural Science Foundation of China (31271082), by the Fundamental Research Fund for the Central Universities, by the Hong Kong Research Grant Council grant 2120298 in Hong Kong, and by US Natural Science Foundation grant BCS\#0350272.

\section{References}

Anglin, J.M. (1993). Vocabulary development: a morphological analysis. Monographs of the Society for Research in Child Development, 58 (10, Serial No. 238), 1-165.

Bauer, D.J., Goldfield, B.A., \& Reznick, J.S. (2002). Alternative approaches to analyzing individual differences in the rate of early vocabulary development. Applied Psycholinguistics, 23 (03), 313-335.

Boscardin, C.K., Muthén, B., Francis, D.J., \& Baker, E.L. (2008). Early identification of reading difficulties using heterogeneous developmental trajectories. Journal of Educational Psychology, 100 (1), 192-208.

Bowey, J.A. (2001). Nonword repetition and young children's receptive vocabulary: a longitudinal study. Applied Psycholinguistics, 22 (3), 441-469.

Chen, X., Anderson, R.C., Li, W., Hao, M., Wu, X., \& Shu, H. (2004). Phonological awareness of bilingual and monolingual Chinese children. Journal of Educational Psychology, 96 (1), 142-151.

Chow, B.W.-Y., McBride-Chang, C., \& Burgess, S. (2005). Phonological processing skills and early reading abilities in
Hong Kong Chinese kindergarteners learning to read English as a second language. Journal of Educational Psychology, 97 (1), 81-87.

Duursma, E., Romero-Contreras, S., Szuber, A., Proctor, P., Snow, C., August, D., \& Calderón, M. (2007). The role of home literacy and language environment on bilinguals' English and Spanish vocabulary development. Applied Psycholinguistics, 28 (1), 171-190.

Fernald, A., \& Marchman, V.A. (2012). Individual differences in lexical processing at 18 months predict vocabulary growth in typically developing and late-talking toddlers. Child Development, 83 (1), 203-222.

Gathercole, S.E., Service, E., Hitch, G.J., Adams, A.M., Martin, A.J (1999). Phonological short-term memory and vocabulary development: further evidence on the nature of the relationship. Applied Cognitive Psychology, 13, 65-77.

Gathercole, S.E., Willis, C.S., Emslie, H., \& Baddeley, A.D. (1992). Phonological memory and vocabulary development during the early school years: a longitudinal study. Developmental Psychology, 28 (5), 887-898.

Ho, C.S.-H., Chan, D.W.-O., Lee, S.-H., Tsang, S.-M., \& Luan, V.H. (2004). Cognitive profiling and preliminary subtyping in Chinese developmental dyslexia. Cognition, 91 (1), 43-75.

Hoff, E. (2003). The specificity of environmental influence: socioeconomic status affects early vocabulary development via maternal speech. Child Development, 74 (5), 1368-1378.

Hurtado, N., Marchman, V.A., \& Fernald, A. (2008). Does input influence uptake? Links between maternal talk, processing speed and vocabulary size in Spanish-learning children. Developmental Science, 11 (6), F31-F39.

Huttenlocher, J., Haight, W., Bryk, A., Seltzer, M., \& Lyons, T. (1991). Early vocabulary growth: relation to language input and gender. Developmental Psychology, 27 (2), 236-248.

Just, M.A., \& Carpenter, P.A. (1992). A capacity theory of comprehension: individual differences in working memory. Psychological Review, 99, 122-149.

Kale, S.H. (1995). Grouping Euroconsumers: a culture-based clustering approach. Journal of International Marketing, 3 (3), 35-48.

Lee, J. (2010). Size matters: early vocabulary as a predictor of language and literacy competence. Applied Psycholinguistics, 32 (01), 69-92.

Lei, L., Pan, J., Liu, H., McBride-Chang, C., Li, H., Zhang, Y., Chen, L., Tardif, T., Liang, W., Zhang, Z., \& Shu, H. (2011). Developmental trajectories of reading development and impairment from ages 3 to 8 years in Chinese children. Journal of Child Psychology and Psychiatry, 52 (2), 212-220.

Lervåg, A., \& Aukrust, V.G. (2010). Vocabulary knowledge is a critical determinant of the difference in reading comprehension growth between first and second language learners. Journal of Child Psychology and Psychiatry, 51 (5), 612-620.

Li, H., Shu, H., McBride-Chang, C., Liu, H., \& Peng, H. (2012). Chinese children's character recognition: visuo-orthographic, phonological processing and morphological skills. Journal of Research in Reading, 35 (3), 287-307. 
Liu, P.D., \& McBride-Chang, C. (2010). What is morphological awareness? Tapping lexical compounding awareness in Chinese third graders. Journal of Educational Psychology, 102 (1), 62-73.

McBride-Chang, C., Cho, J.R., Liu, H., Wagner, R.K., Shu, H., Zhou, A., Cheuk, C.S., \& Muse, A. (2005a). Changing models across cultures: associations of phonological awareness and morphological structure awareness with vocabulary and word recognition in second graders from Beijing, Hong Kong, Korea, and the United States. Journal of Experimental Child Psychology, 92 (2), 140-160.

McBride-Chang, C., Chow, B.W.Y., Zhong, Y., Burgess, S., \& Hayward, W.G. (2005b). Chinese character acquisition and visual skills in two Chinese scripts. Reading and Writing, 18 (2), 99-128.

McBride-Chang, C., Liu, P.D., Wong, T., Wong, A., \& Shu, H. (2011). Specific reading difficulties in Chinese, English, or both: longitudinal markers of phonological awareness, morphological awareness, and RAN in Hong Kong Chinese children. Journal of Learning Disabilities, 45 (6), 503-514.

McBride-Chang, C., Shu, H., Zhou, A., Wat, C.P., \& Wagner, R.K. (2003). Morphological awareness uniquely predicts young children's Chinese character recognition. Journal of Educational Psychology, 95 (4), 743-751.

McBride-Chang, C., \& Suk-Han Ho, C. (2005). Predictors of beginning reading in Chinese and English: a 2-year longitudinal study of Chinese kindergartners. Scientific Studies of Reading, 9 (2), 117-144.

McBride-Chang, C., Tardif, T., Cho, J.-R., Shu, H.U.A., Fletcher, P., Stokes, S.F., Wong, A., \& Leung, K. (2008). What's in a word? Morphological awareness and vocabulary knowledge in three languages. Applied Psycholinguistics, 29 (03), 437-462.

McBride-Chang, C., Wagner, R.K., Muse, A., Chow, B., \& Shu, H. (2005c). The role of morphological awareness in children's vocabulary acquisition in English. Applied Psycholinguistics, 26 (3), 415-435.

Moll, K., Fussenegger, B., Willburger, E., \& Landerl, K. (2009). RAN is not a measure of orthographic processing: evidence from the asymmetric German orthography. Scientific Studies of Reading, 13 (1), 1-25.

Nation, K., Cocksey, J., Taylor, J.S.H., \& Bishop, D.V.M. (2010). A longitudinal investigation of early reading and language skills in children with poor reading comprehension. Journal of Child Psychology and Psychiatry, 51, 1031-1039.

Pan, B.A., Rowe, M.L., Singer, J.D., \& Snow, C.E. (2005). Maternal correlates of growth in toddler vocabulary production in low-income families. Child Development, 76 (4), 763-782.

Pan, J., McBride-Chang, C., Shu, H., Liu, H., Zhang, Y., \& Li, H. (2011). What is in the naming? A 5-year longitudinal study of early rapid naming and phonological sensitivity in relation to subsequent reading skills in both native Chinese and English as a second language. Journal of Educational Psychology, 103 (4), 897.

Perfetti, C. (2009). Decoding, vocabulary, and comprehension. In M. McKeown \& L. Kucan (Eds.), Bringing reading research to life (pp. 291-303). New York and London: Guilford Press.

Perfetti, C.A., Landi, N., \& Oakhill, J. (2005). The acquisition of reading comprehension skill. In M.J. Snowling \& C. Hulme (Eds.), The science of reading: A handbook (pp. 227247). Oxford: Blackwell.

Raven, J.C., Court, J.H., \& Raven, J. (1996). Standard progressive matrices. Oxford: Oxford Psychologists Press.

Rogosa, D., Brandt, D., \& Zimowski, M. (1982). A growth curve approach to the measurement of change. Psychological Bulletin, 92 (3), 726-748.

Rowe, M.L., \& Goldin-Meadow, S. (2009). Differences in early gesture explain SES disparities in child vocabulary size at school entry. Science, 323 (5916), 951-953.

Rowe, M.L., Raudenbush, S.W., \& Goldin-Meadow, S. (2012). The pace of vocabulary growth helps predict later vocabulary skill. Child Development, 83 (2), 508-525.

Shu, H., \& Anderson, R.C. (1998). Learning to read Chinese: the development of metalinguistic awareness. In J. Wang, A.W. Inhoff \& H.-C. Chen (Eds.), Reading Chinese script: A cognitive analysis (pp. 1-18). Mahwah, NJ: Lawrence Erlbaum.

Shu, H., Chen, X., Anderson, R.C., Wu, N., \& Xuan, Y. (2003). Properties of school Chinese: implications for learning to read. Child Development, 74 (1), 27-47.

Shu, H., McBride-Chang, C., Wu, S., \& Liu, H. (2006). Understanding Chinese developmental dyslexia: morphological awareness as a core cognitive construct. Journal of Educational Psychology, 98 (1), 122-133.

Singer, J.D., \& Willett, J.B. (2003). Applied longitudinal data analysis: Modeling change and event occurrence. New York: Oxford University Press.

Stokes, S.F., \& Klee, T. (2009). Factors that influence vocabulary development in two-year-old children. Journal of Child Psychology and Psychiatry, 50 (4), 498-505.

Storch, S.A., \& Whitehurst, G.J. (2002). Oral language and code-related precursors to reading: evidence from a longitudinal structural model. Developmental Psychology, 38 (6), 934-947.

Tardif, T., Fletcher, P., Zhang, Z.X., \& Liang, W.L. (2008). The Chinese Communicative Development Inventory (Putonghua and Cantonese versions): Manual, forms, and norms. Beijing: Peking University Medical Press.

Tataryn, D., \& Chochinov, H.M. (2002). Predicting the trajectory of will to live in terminally ill patients. Psychosomatics, 43 (5), 370-377.

Thorndike, R.L., Hagen, E.P., \& Sattler, J.M. (1986). The Stanford-Binet intelligence scale: Guide for administering and scoring. Itasca, IL: Riverside Publishing Company.

Tong, X., McBride-Chang, C., Shu, H., \& Wong, A.M.Y. (2009). Morphological awareness, orthographic knowledge, and spelling errors: keys to understanding early Chinese literacy acquisition. Scientific Studies of Reading, 13 (5), 426-452.

Vagh, S.B., Pan, B.A., \& Mancilla-Martinez, J. (2009). Measuring growth in bilingual and monolingual children's English productive vocabulary development: the utility of combining parent and teacher report. Child Development, 80 (5), 1545-1563. 
Verhoeven, L., van Leeuwe, J., \& Vermeer, A. (2011). Vocabulary growth and reading development across the elementary school years. Scientific Studies of Reading, 15 (1), 8-25.

Zhang, J., McBride-Chang, C., Wong, A.M.-Y., Tardif, T., Shu, H., \& Zhang, Y. (2013). Longitudinal correlates of reading comprehension difficulties in Chinese children. Reading and Writing, 1-21.
Zhang, Y., Tardif, T., Shu, H., Li, H., Liu, H., McBride-Chang, C., Liang, W., \& Zhang, Z. (2013). Phonological skills and vocabulary knowledge mediate socioeconomic status effects in predicting reading outcomes for Chinese children. Developmental Psychology, 49 (4), 665-671.

Received: 4 March 2013

Accepted: 7 March 2014 